\title{
Sterben: Schicksal, Selbstbestimmung oder beides?
}

\author{
Anita Lanz \\ IPS-Onkologie-Anästhesie-Pflegefachfrau
}

Eigentlich bin ich seit Ende Juni, vier Jahre nach erreichtem Pensionsalter, im Ruhestand. Aber noch immer erreichen mich Hilferufe, die ich nicht überhören kann. Von einem, der mich vor ganz neue Herausforderungen stellte, sei hier die Rede. Bei Anna, Jahrgang 1959, wurde im Sommer 2012 die Diagnose eines fortgeschrittenen Mammakarzinoms gestellt, als sie einen Arzt aufsuchte wegen eines Tumors in der Brust, der schon exulzeriert war. Es folgte eine schulmedizinische Therapie mit Chemotherapien und Bestrahlungen in verschiedenen renommierten Spitälern. Im Januar 2013 besuchte sie auf Empfehlung eines Bekannten einen geheilten Krebspatienten im Appenzellischen. Dieser verhielt sich korrekt, erzählte ihr seine Geschichte, die seine Geschichte sei, wie er immer wieder betonte, und dass er persönlich gut gefahren sei mit dem Buch Die neue Anti-Krebs-Ernährung - wie Sie das Krebs-Gen stoppen. Er verlangte kein Honorar für die Konsultation, er bot aber Anna an, sie zu unterstützen, wenn sie sich anders ernähren wolle. Eigentlich waren noch zwei Chemotherapien vorgesehen, aber nach Abschluss der ersten Chemo wechselte Anna zu einer homöopathischen Behandlung. Ein anderer Bekannter empfahl Anna einen Geistheiler.

\section{Säfte, Carbon und Kaffee-Einläufe}

Damit war der Anfang einer traurigen Geschichte gegeben, die in ähnlicher Form vermutlich öfter vorkommt, als wir ahnen. Ich habe während meiner Tätigkeit in der Onko-Spitex meine Patienten immer gefragt, ob sie alternative Therapien in Ergänzung zu ihren schulmedizinischen Therapien verwendeten. Dabei habe ich ganz interessante Antworten bekommen, und vor allem habe ich vorher nicht geahnt, was in diesem Bereich alles angeboten wird.

Anna fasste grosses Vertrauen in den Geistheiler und liess sich für vier Wochen in seinem Wohnort einquartieren. Kosten ohne Logis 16400 Franken. Sie folgte einem genauen Therapieplan. Das Essen bestand praktisch nur aus verschiedenen Säften. Dazu gab es Carbon und Cayenne und täglich dreimal Kaffee-Einläufe, manchmal mit Zusatz von Betadine ${ }^{\circledR}$. In der Zwischenzeit entwickelte sich eine riesige Wunde mit nach aussen wuchernder Tumormasse. Sie wurde von der Krankenschwester mit einer Mischung aus Lipoderm $^{\circledast}$, Jod und Leinöl behandelt. Annas Freundin beschloss, die Schwerkranke vom Geistheiler wegzuholen und sie bei sich zu Hause bis zu ihrem Tod zu pflegen. Gleichzeitig nahm sie mit mir Kontakt auf. Meine erste Begegnung mit Anna war nicht einfach. Sie war stark abgemagert und kachektisch. Sie liess mich sofort verstehen, dass sie keine Medikamente schulmedizinischer Herkunft einnehmen werde. Ich sah ihr schmerzgeplagtes Gesicht und fragte nach der Schmerzstärke gemäss VAS-Skala ${ }^{1}$. Sie gab, wie ich schon vermutete, mit 10 die stärksten vorstellbaren Schmerzen an. Ich suchte in ihrer Medikamentenschachtel und fand Novalgin $^{\circledR}$, wovon ich kurzerhand zwei Tabletten zerkleinerte und sie ihr mit etwas Wasser auf einem Löffel in den Mund schob. Sie schluckte wortlos. Kurz darauf jammerte sie über Übelkeit, Magenschmerzen, Brennen und Aufstossen. Ich setzte mich sofort ins Auto und holte bei ihrem Hausarzt - zum Glück ein Arzt mit Selbstdispensation - Esomep ${ }^{\circledR}$, einen Magenschoner. Auch diese Tablette wurde zerkleinert und Anna verabreicht. Schon am Mittag ging es ihr besser und sie konnte etwas essen. Am nächsten Tag schluckte sie die Tablette freiwillig. Wir alle atmeten auf, aber Anna blieb, obwohl es ihr etwas besser ging, bei ihrem schon vorher gefassten Entschluss, mit Exit zu sterben.

\section{Der Entschluss: Anna stirbt mit Exit}

Ich eröffnete ihr alle Möglichkeiten, die die Palliativmedizin zu bieten hat, und erklärte, dass wir in unserem Spital eine gute Palliativmedizin hätten und dass ich sie dort bis zum Tod betreuen würde. Anna blieb bei ihrem Entschluss. Die Schmerzen und die riesige Wundfläche wurden immer mehr zum Problem. Die Wunde blutete bei jedem Verbandwechsel und Annas Gesicht war gezeichnet von Angst und Schmerzen. Ich besorgte Morphintropfen, Durogesic ${ }^{\circledR}$, Haldol $^{\circledR}$ und Temesta ${ }^{\circledR}$ beim Hausarzt. Ich erläuterte ihr, dass es auch für uns alle schwer war, dieses Leiden anzuschauen, und dass die Möglichkeit bestand, mit Pflaster die Schmerzen zu nehmen. Mein Angebot, mit einem halben Pflaster anzufangen und am nächsten Tag dann eventuell die 
zweite Hälfte zu kleben, nahm sie an. Am nächsten Tag durfte die Spitex den zweiten Teil aufkleben. Am Tag vor ihrem Tod willigte sie in eine grosszügigere Verwendung des Pflasters ein. Nach und nach verabreichten wir zusätzlich Morphintropfen und schliesslich auch Temesta $^{\circledR}$ und Haldol ${ }^{\circledR}$. Die Situation beruhigte sich. Der von ihr getrennt lebende Vater ihres Sohnes, eine Freundin und ihre Brüder durften, obwohl sie dies vorher abgelehnt hatte, vorbeikommen, um Abschied zu nehmen. Eine Beraterin von Exit und auch die Exitärztin besuchten Anna, verschrieben das Rezept. Das Sterbedatum und die genaue Zeit wurden festgelegt: Am Samstag um 10 Uhr morgens. Anna bestimmte ihren Todestag so, dass er auf den Geburtstag ihres einzigen Sohnes fiel. Statt seinen Geburtstag zu feiern, sollte er also den Tod seiner Mutter erleben. Um diesen Sohn zu unterstützen, sprang ich weit über meinen Schatten. Obwohl ich mich grundsätzlich von Exit distanziere, fühlte ich die Verantwortung für den Sohn und Annas Freundin. Ich beschloss, ihnen beim freiwilligen Sterben der Todkranken beizustehen. Das war etwas, das ich bis dahin mit meinen ethischen und moralischen Werten nicht hatte vereinbaren können. Manchmal siegt der Instinkt über Ethik und Moral. Das Muttergefühl ist wohl eines der unantastbarsten Gefühle. Es lässt sich schwer dem Verstand unterordnen. Zum Glück ist der starke innere Wunsch, die Schwächeren zu schützen, bei vielen von uns immer noch vorhanden.

Am Samstag um neun Uhr morgens begab ich mich auf den Weg zu Anna. Ich fühlte mich nicht wohl dabei, aber ich musste an den Sohn denken. Ich erinnerte mich, wie schrecklich es für meine jüngste Tochter gewesen war, als der Grossvater an ihrem achtzehnten Geburtstag unerwartet verstarb.

Am Tag vorher hatte ich dem Sohn und der Freundin den genauen Ablauf nach dem Eintritt des Todes geschildert und ihnen geraten, die Bestatterin anzurufen, damit sie die Polizei und den Bezirksarzt vorbereiten können, um so unnötige Wartezeiten zu vermeiden.

\section{Der Kreislauf schliesst sich}

Die zwei Begleiter von Exit erschienen und wählten von den Anwesenden ausgerechnet mich aus, um das Vorgehen zu erklären. Anna hatte aber gewünscht, dass nur ihre Freundin und der Sohn anwesend sein sollten. Die Exitbegleiter verhielten sich sehr korrekt und freundlich. Ich war also nicht dabei, als der Anästhesist die Infusion legte. Aber Annas Freundin erzählte mir später, Anna, die kaum mehr reden konnte, habe ihn angeschaut und habe gesagt: «In Sie habe ich Vertrauen.» Anna musste mehrere Versuche unternehmen, bis sie den Hahn der Infusion öffnen konnte, da sie sehr schwach war. Nach einer Weile funktionierte es aber und Anna verliess kurz darauf, ein paar Minuten vor elf, diese Welt. Um elf Uhr vor 26 Jahren war ihr einziger Sohn geboren.

Der Vater, die Freundin des Sohnes und ich warteten im Garten. Die junge Frau litt sehr unter der Situation. Ich erklärte ihr, wie auch schon vorher dem Sohn, dass der grosse Schmerz des Verlustes nicht das Einzige sei, dass auch ein Kreislauf von Geburt und Tod sich jetzt schliessen würde. Dass der Tod vielleicht auch eine Form der Geburt der Seele in eine andere Welt sei.

Als der Tod eingetreten war, blieb ich noch eine Weile neben der toten Anna sitzen und schaute ihre gelösten Gesichtszüge an. Sie wirkte ganz entspannt. Das Leiden hatte ein Ende gefunden. Für Anna war dies der letzte selbstbestimmte Entscheid gewesen, den sie in ihrem Leben getroffen hatte. Annas Freundin hatte gezeigt, was wirkliche Freundschaft sein kann, und Annas Sohn hatte mit seinem Vater im kleinen Familienkreis von seiner Mutter Abschied nehmen können.

\section{Offen für verschiedene Formen des Sterbens}

Ich brachte den Exit-Mitarbeiter nach Annas Tod zum Bahnhof. Seine Aufgabe an diesem Tag hätte ich an seiner Stelle zwar nicht übernommen, aber ich sah, dass er einen ganz anderen Auftrag zu erfüllen hat als ich als Palliativschwester. Warum sollen wir einander nicht Respekt erweisen? Anna hat aus freiem Willen diesen Weg, der heute Menschen offensteht, gewählt, und wir haben kein Recht, diesen Entscheid nach unserer persönlichen Meinung zu beurteilen.

Das Gesicht des Todes hat sich in den letzten Jahren stark gewandelt. Es gibt immer mehr Beteiligte rund um das Sterben: Pflegende aus Spitälern, von der Spitex, Palliativpflegefachfrauen, Physiotherapeuten, Hausärzte, Onkologen, Palliativärzte, Radioonkologen, Psychologen, Psychoonkologen, Psychiater, Sozialarbeiter, Seelsorger, Geist- und andere Heiler. Das Positive daran ist, dass Menschen nicht mehr dem Leiden ausgeliefert sind und immer länger am Leben bleiben können. Schwierig wird es, wenn der Patient alles ablehnt, keine Therapien mehr will und sterben möchte. Da lässt sich nicht immer jemand finden, der den Mut hat, mit ihm ganz offen über Tod und Sterben zu reden, jemand, der seinen Wunsch respektiert. Es ist einfacher, eine Therapie vorzuschlagen, auch wenn man weiss, dass der Erfolg gering ist. Und manchmal sogar ein natürliches, ruhiges Sterben erschwert.

Nicht nur das Leben, auch das Sterben kann, wie ich aus langjähriger Erfahrung weiss, etwas sehr Bewegendes und Ergreifendes sein. Es lohnt sich, ihm mit offenen Augen und anteilnehmendem Blick zu begegnen. 